\title{
Performance Analysis of Ad hoc Routing Protocols over VANETs
}

\author{
Paramjit Waraich \\ PhD Research Scholar, \\ CSE Deptt.,MMEC, \\ M. M. University, Ambala, India
}

\author{
Neera Batra, $\mathrm{PhD}$ \\ Associate Professor \\ CSE Deptt., MMEC, \\ M. M. University, Ambala, India
}

\begin{abstract}
VANET is a large scale adhoc networks that has several performance issues i.e. Quality of Services, Security, Dynamic Topology and Mobile environment etc. Researchers have developed various solutions for these issues but each has its own limitations. Issues related to QoS are most critical because end user suffers a lot due to low grade data transmission. Network performance also depends upon the behavior of the routing protocol. So there is need to develop a relationship between QoS, network performance and the behavior of routing protocols. In this paper, performance analysis of various routing protocols (AODV, DSR, GPSR, LAR and OLSR) has been analyzed to provide the QoS over VANETs.
\end{abstract}

\section{Keywords}

VANET, AODV, DSR, GPSR, LAR and OLSR, QoS

\section{INTRODUCTION}

VANET based applications operate in real time environment and it is essential to maintain the level of QoS and QoE for end user. As per the application domain, values for each constraint is changed. For multimedia streaming over VANET, routing protocol must deliver the contents with any Delay/Jitter, for military operations, security is prime issue. So there should be a provision which can maintain the QoS for each domain [5 ]. QoS suffers from the following factors:
a. Dynamic Location update
b. Environmental Impact over channel
c. Channel Interference
d. Velocity
e. Contention
f. Congestion [15][16][18]

\subsection{Intelligent Transportation System (ITS)}

ITS offers automatic application synchronization with vehicles. ITS based application can dynamically update the different type of messages i.e. Safety Message, Traffic Condition, Road Condition, Accident Alerts and Road Jam Alerts etc. Following are the type of VANET based Communication: [17]

a. Vehicle to Infrastructure (V2I)

In V2I, nodes interact with road side units (RSU) for data exchange.

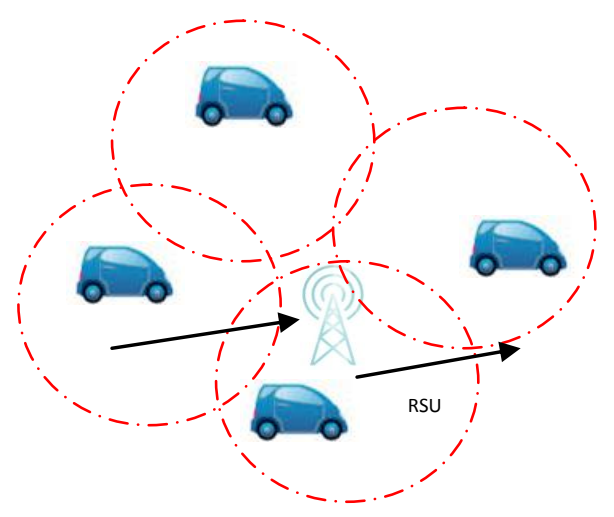

Fig 1: V2I Communication over VANET

b. Vehicle to Vehicle (V2V)

In $\mathrm{V} 2 \mathrm{~V}$, nodes interact with other nodes and routing information is updated frequently through dynamic topology.

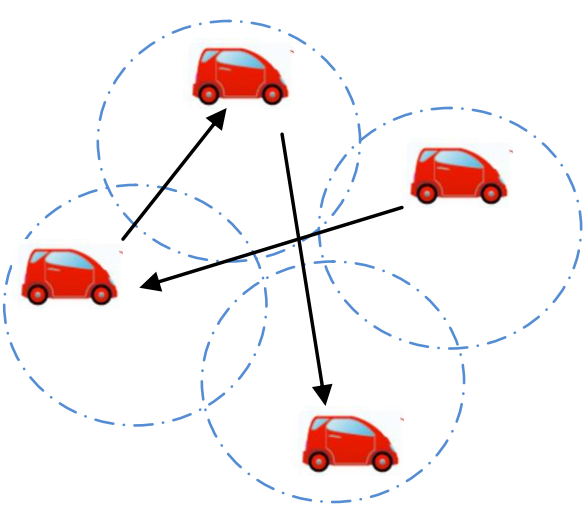

Fig 2: V2V Communication over VANET

\section{RELATED WORK}

ITS based applications must fulfill the QoS constraints. Researchers have developed various solutions to meet the end user requirements. This section explores the contribution made by them. H. El Ajaltouni [1] et al. proposed a solution for VANET which can sense the channel before transmission and selects optimal multipath having less interference, called Multichannel QoS Cognitive MAC (MQOG). MQOG uses dynamic channel allocation methods based on unlicensed bands under the constraints of QoS. Simulation results show that it improves PDR, Throughput and minimize the Delay. 


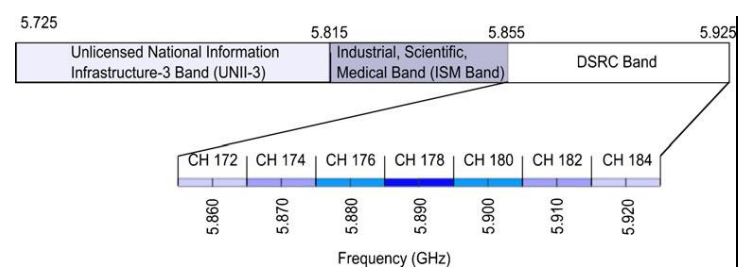

Fig 3: Unlicensed Bands [1]

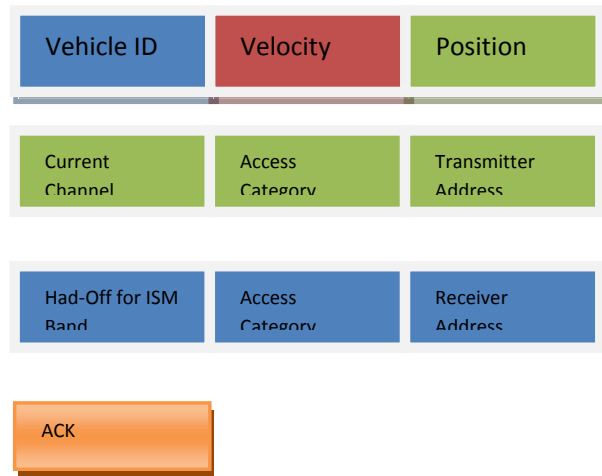

Fig 4: Control frames used on dedicated control channel [1]

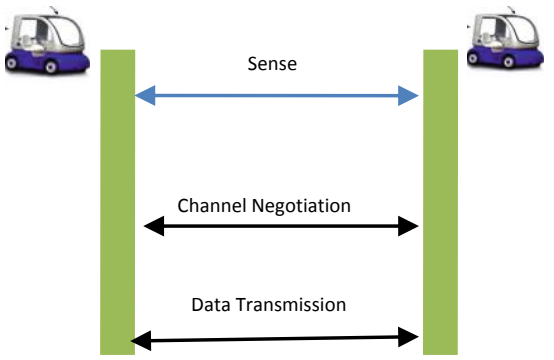

Fig 5: Channel Negotiation [1]

J. Mun-Yee Lim [2] et.al, developed a Heuristic and Adaptive Fuzzy Logic Scheme, called HaFL which can regulate the size of contention window as per the traffic conditions and available transmission power. It analyzes the different parameters at different layers i.e. Queue length \& collision rate at MAC layer and SINR from PHY layer to identify congestion status. On the basis of collected statistics, it regulates the size of contention window and transmission power using fuzzy logic. Simulation results show that if SINR increase, it may lead to hidden terminal problem and if it is lowest level, it may degrade the transmission ratio. HaFL performs well under high mobility constraints and maintains the ratio of Throughput, PDR and Delay.

Lin Zhang [3] et al. proposed a service oriented solution; called Mixed-Service-Mobility model (MSM) for VANET based applications. It supports both delay-tolerant and real time services. It is able to update the size of contention window according to the variations in vehicle's velocity and tries to maintain at its lowest level. Simulation results show that using MAC 802.11p, it supports delay tolerance and channel fairness for real time applications i.e. Video/Voice etc. Current solution supports the QoS only but can be extended to provide the provision of Quality of Experience (QoE)/Quality of Perception (QoP) metrics.

Guangyu Li [4] et al. proposed a solution based on ACO, called Intersection based routing with Quality of Service support over VANET (IRQV). ACO provides the optimal paths based on pheromone value at the intersections and local road segments ensure the probability of connectivity with minimum delay. Simulation results show that it outperforms the GSR/CAR protocol.

V.Vijayakumara [5] et al. investigated the requirements of QoS enabled communication over VANET under various constraints, i.e. Throughput, Delay, efficiency, PDR, Message Priority and service disruption time etc.

Table .1 QoS Constraints for Protocols

QoS OLSR IPTV Collaborative EDCA

parameter(s)

protocol

\begin{tabular}{|c|c|c|c|c|}
\hline Delay & $\mathrm{x}$ & $\mathrm{x}$ & $\mathrm{x}$ & $\mathrm{x}$ \\
\hline Mobility & $\mathrm{x}$ & & & \\
\hline Jitter & & $X$ & & \\
\hline PSNR & & $X$ & & \\
\hline $\begin{array}{l}\text { Packet Loss } \\
\text { Ratio }\end{array}$ & & $X$ & & \\
\hline PDR & $\mathrm{X}$ & $\mathrm{X}$ & & \\
\hline Throughput & & $X$ & & $\mathrm{x}$ \\
\hline Bandwidth & & $\mathrm{X}$ & & \\
\hline
\end{tabular}

It can be observed that value of each parameter varies w.r.t. the protocol and traffic type. This survey explores the various protocols I.e. OLSR, IPTV, Collaborative protocol and their relevant traffic types ( Video/ Multimedia/Internet Traffic).This study can be utilized to develop a QoS aware routing protocol for VANET based application.

M. E. A. Fekair [6] et.al. presented a QoS aware protocol for VANETs. It uses clustering algorithm to collect routing data to fulfill QoS requirements and uses ACO to find the optimal paths on the basis of gathered information. Cluster head is elected on the basis of various QoS parameters i.e. Link stability, available bandwidth, Delay and Jitter etc.

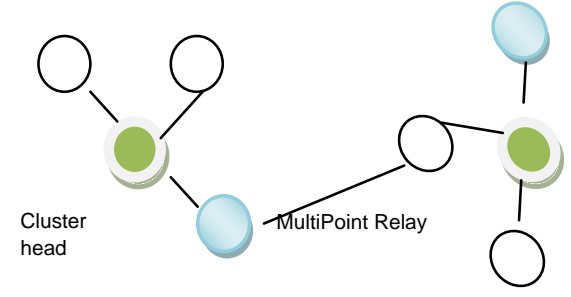

Fig 6: Clusters

Simulation results show that it can find optimal paths and satisfy the various QoS constraints. Current research work can be extended to provide the support for real time applications.

A. El Khatib [7] et al. explored the malicious behavior of intermediate nodes under the constraints of mobility in VANET and proposed a QoS-OLSR solution based on neural networks. Due to node misbehavior, links may be frequently broken and network performance may be degraded. It is quite complex to detect this event. Some detection methods can identify this misbehavior and can raise alarms which may be 
false. Proposed scheme can learn from its experience and can reduce the ratio of false alarms and simulation results show that it enhances the detection probability and minimizes the false alarm rate.

G. el m. Zhioua [8] et al. developed a solution to select a gateway from VANET to LTE networks. It selects Cluster head as gateway on the basis of various constraints i.e. data traffic density, strength of wireless links, node velocity, link stability \& life time etc.

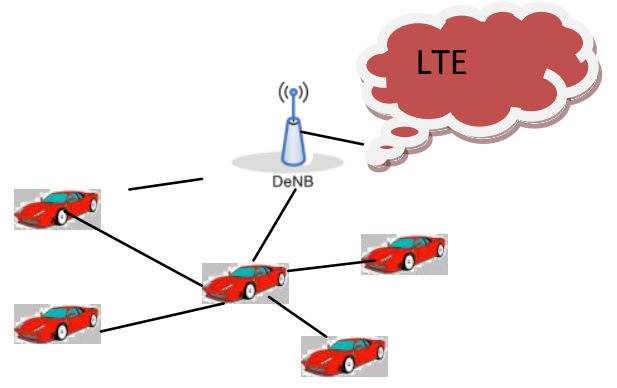

Fig 7: Gateway Selection

Simulation results show its efficiency in terms of gateway selection on the basis of cluster density and node's velocity.

M. H. Eiza [9] et al. presented a method for secure routing using ACO algorithm which can estimates the optimal paths and secure paths based on VANET-oriented Evolving Graph (VoEG) model. It is used to verify the control messages transmission between vehicles. Simulation results show that network performance suffers from extra control overhead due to proposed scheme but it provides most reliable routing paths under QoS constraints (PDR. MoS) for Voice stream.

G. Rizzo [10] et al. invetigated the V2I based communication and the support for Software Defined Networks (SDN), Content Centric Networks (CCN) to fullfill the QoS requirements. $\mathrm{CCN}$ considers the content delivery irrespective to user loction. Mobility support for Sender/Reciver is monitered by routing mechanisms.CCN delas with hetrogenious networks so it is difficult to ensure the QoS for large scale geogarhical networks. SDN ensures the QoS content delivery but its reliability depends upon the Road Side Unit (RSU) and is also suffers from resource managent and limited capabilities of infrastructure being used.

A. Ennaciri [11] et al. did a performance analysis of AODV protocol using H.264 library under the constraints of QoS and Mobility. Simulation results show that performance of network depends upon various factors, i.e. Network Size, Mobility and Bit Rate etc. Quality of Video stream suffers from compression methods, Delay and Jitter. Packet Loss occurs due to dynamic topology.

[12] J. Joshi [12] et al. considered the issues related to the safety of drivers/passangers on the road and introdcued the concept of Intelligent Transportation System by developing a protocol which can switch the Lane automatically on the basis of various mobility constraints, i.e. current speed and the gap between vehicles etc. Mobility pattern for NS-2 is generated using SUMO and MOVE applications and simulation shows its accuracy using AODV/GPSR protocols and its real time implementation indicates that performace and accuracy can be achieved using GPS.

X. L. Huang [13] et al. focused on the extra control over head introduced by the broadcasting of safety messages. Proposed scheme can detect the idle channel conditions and can utilize the available spectrum for transmission of safety messages periodically, to avoid the congestion.

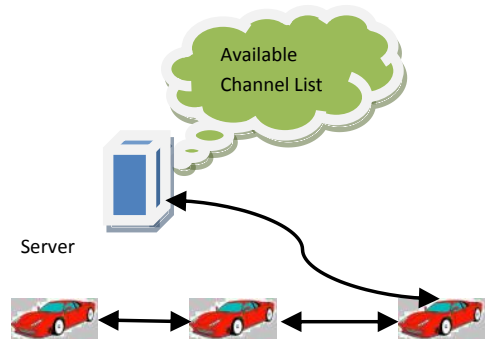

Fig 8: Sharing of Available Channel List

Simulation results show its performance in terms of accuracy of prediction, detection of spectrum, radio of false alarms etc. Proposed work can be extended to detect the channel interference to provide the fair channel allocation support over VANETs.

Che-Yu Chang [14] et al. proposed a QoS solution for V2V based communication, called Earliest Deadline First based Carrier Sense Multiple Access (EDFCSMA). It can dynamically regulate the priorities of real time traffic and avoid the collision over channel using admission control policy which offers optimal medium access to a particular channel. Proposed work can be extended for multi-hop VANETs.

\section{SIMULATION CONFIGURATION}

Table 2. Simulation parameters

\begin{tabular}{|c|c|}
\hline Simulation Parameters & $\begin{array}{c}\text { Parameter Values } \\
\text { AODV, DSR, OLSR, } \\
\text { ZRP, GPSR, LAR }\end{array}$ \\
\hline Routing Protocol(s) & $4000 x 4000$ \\
\hline Terrain & 90 \\
\hline Node Density & MAC 802.11 P \\
\hline MAC Protocol & CBR \\
\hline Propagation Model & Nakagami Fading Model \\
\hline Traffic Type & 0.1 seconds \\
\hline Packet Size & 10 seconds \\
\hline Sampling Interval & NS-2.35 \\
\hline Simulation Time & SUMO 0.21.0 \\
\hline Network Simulator & \\
\hline Traffic Simulator &
\end{tabular}

Steps for MAP generation and export

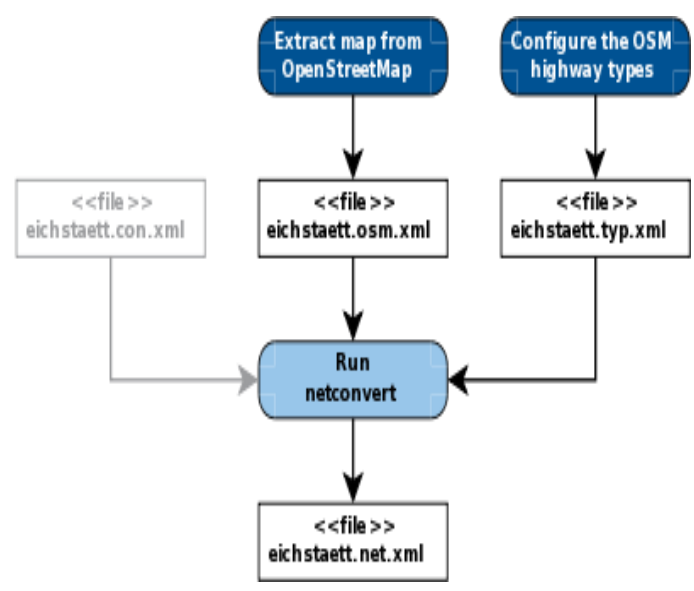

Fig 9 : MAP generation [19] 
Table 3.0 SUMO Trace File Generation

\begin{tabular}{|c|c|}
\hline S.No. & $\begin{array}{c}\text { Convert MAP to SUMO } \\
\text { Network }\end{array}$ \\
\hline 1. & netconvert --xml-type-files osm- \\
& vanet.typ.xml --osm-files \\
& vanet.osm --output-file \\
& vanet.net.xml \\
\hline
\end{tabular}

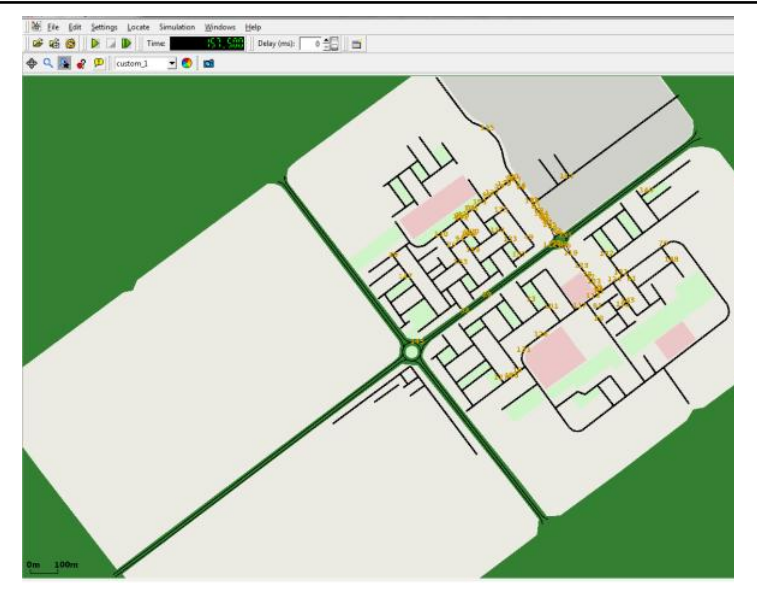

Fig 9.1: MAP exported from www. openstreetmap.com.

We generated SUMO vehicle mobility traces for NS-2. [19][20][21][22]

Table 3.1. SUMO Trace File Generation

\begin{tabular}{|c|c|}
\hline S.No. & Steps to generate SUMO Trace File \\
\hline 1. & sumo -c vanet.sumocfg \\
\hline 2. & $\begin{array}{c}\text { sumo -c vanet.sumocfg --fcd-output } \\
\text { vanetTrace.xml }\end{array}$ \\
\hline 3. & $\begin{array}{c}\text { Convert SUMO trace file to NS-2 file compatible } \\
\text { format }\end{array}$ \\
\hline 4. & $\begin{array}{c}\text { traceExporter.py --fcd-input vanetTrace.xml -- } \\
\text { ns2mobility-output ns2mobility.tcl }\end{array}$ \\
\hline 5. & $\begin{array}{c}\text { traceExporter.py --fcd-input vanetTrace.xml -- } \\
\text { ns2activity-output ns2mobility.tcl }\end{array}$ \\
\hline 6. & $\begin{array}{c}\text { traceExporter.py --fcd-input vanetTrace.xml -- } \\
\text { ns2config-output ns2mobility.tcl }\end{array}$ \\
\hline
\end{tabular}

\section{PERFORMANCE ANALYSIS}

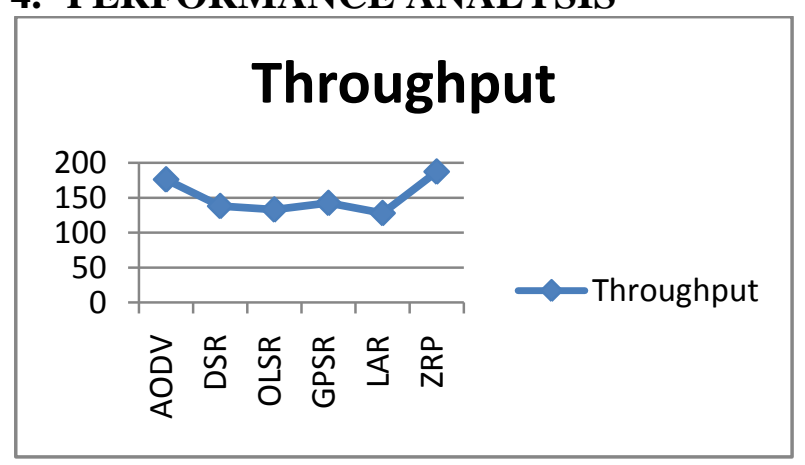

Fig10: Throughput

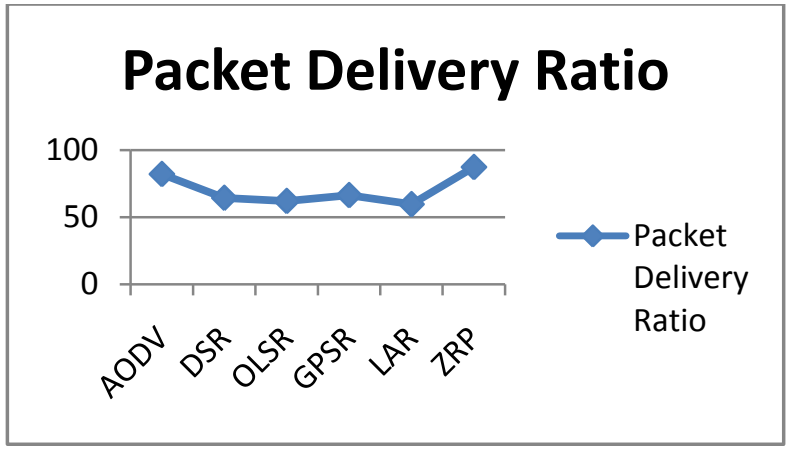

Fig 11: Packet Delivery Ratio

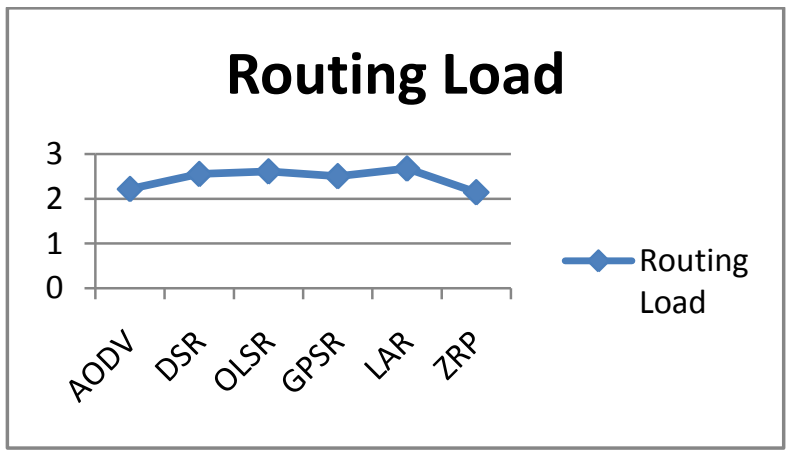

Fig12: Routing Load

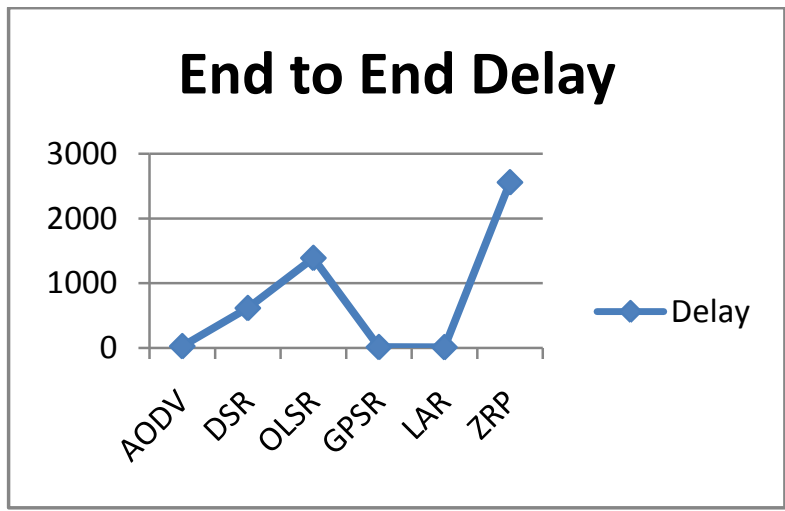

Fig13 End-to-End Delay

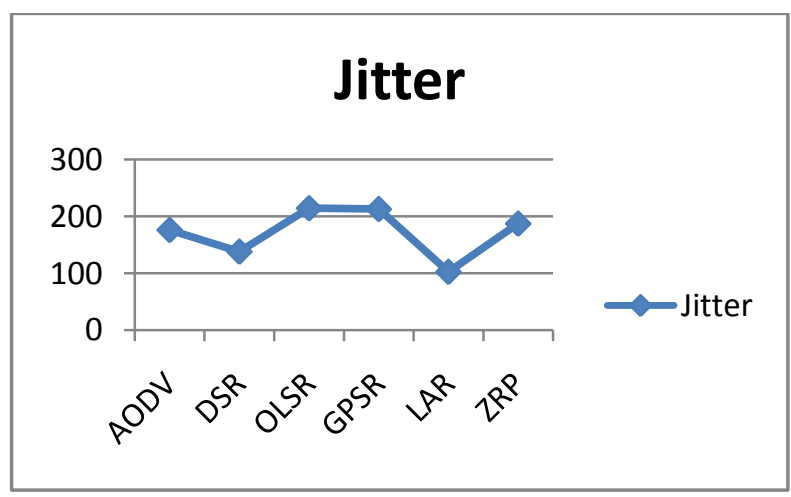

Fig14 Jitter 
Table 4 Performance Analysis

\begin{tabular}{|c|c|c|c|c|c|}
\hline $\begin{array}{c}\text { Proto } \\
\text { cols }\end{array}$ & $\begin{array}{c}\text { Throug } \\
\text { hput } \\
\text { (bps) }\end{array}$ & PDR & $\begin{array}{c}\text { Routing } \\
\text { Load }\end{array}$ & $\begin{array}{c}\text { E2e } \\
\text { Delay } \\
(\mathbf{m s})\end{array}$ & $\begin{array}{c}\text { Jitte } \\
\mathbf{r}\end{array}$ \\
\hline AODV & 176.1 & $\begin{array}{c}82.1745 \\
217\end{array}$ & $\begin{array}{c}2.21692 \\
2203\end{array}$ & 24.8829 & $\begin{array}{c}176 . \\
13\end{array}$ \\
\hline DSR & 138 & $\begin{array}{c}64.3957 \\
0695\end{array}$ & $\begin{array}{c}2.55289 \\
8551\end{array}$ & 614.455 & $\begin{array}{c}138 . \\
06\end{array}$ \\
\hline OLSR & 133 & $\begin{array}{c}62.0625 \\
2916\end{array}$ & $\begin{array}{c}2.61127 \\
8195\end{array}$ & 1388.81 & $\begin{array}{c}214 . \\
41\end{array}$ \\
\hline GPSR & 142.4 & $\begin{array}{c}66.4489 \\
0341\end{array}$ & $\begin{array}{c}2.50491 \\
573\end{array}$ & 11.0485 & $\begin{array}{c}212 . \\
91\end{array}$ \\
\hline LAR & 127.9 & $\begin{array}{c}59.6826 \\
8782\end{array}$ & $\begin{array}{c}2.67552 \\
7756\end{array}$ & 9.2904 & $\begin{array}{c}102 . \\
53\end{array}$ \\
\hline ZRP & 187.3 & 3994 & 3764 & 2558.32 & 32 \\
\hline
\end{tabular}

AODV and ZRP have the highest Throughput/PDR followed by GPSR, DSR, OLSR and LAR. ZRP has the minimum Routing Load and LAR has the highest whereas OLSR, DSR and GPSR has higher Routing Load as compared to AODV and ZRP. LAR, GPSR and AODV have minimum offers minimum End to End Delay and ZRP has the highest Delay followed by OLSR and DSR. LAR has minimum Jitter followed by DSR and AODV. GPSR and OLSR has the highest Jitter followed by ZRP.

\section{CONCLUSION}

In this paper, we did a performance analysis of various ad hoc routing protocols i.e. Reactive, Proactive, Hybrid and Geographical etc. under various QoS constraints i.e. Throughput, Packet Delivery Ratio, End-to-End Delay, Jitter and Routing Load etc.

In case of reactive protocols, AODV has the highest Throughput/PDR as compared to DSR. AODV also offers minimum Routing Load and Delay as compared to DSR which has the minimum Jitter (as compared to AODV).

In case of proactive protocol, OLSR did not perform well as compared to reactive and hybrid routing protocols because it is table driven protocol and cannot adopt the dynamic changes in network topology which generates extra control overhead due to frequent updates in routing information. It has the lowest Throughput/PDR followed by LAR. OLSR has highest End-to-End Delay followed by ZRP. Jitter value of OLSR is approx. similar to GPSR, which is highest as compared to other protocols.

In case of Hybrid routing protocol, ZRP offers highest Throughput/PDR followed by reactive, proactive and geographical routing protocols, but at the cost of End-to-End Delay which is the highest. Its Jitter value is approx. similar to AODV. ZRP uses both approaches i.e. reactive and proactive. Inter-zone communication uses reactive approach and Intra-zone uses proactive approach. So flow of control packets from both zones may lead to the unnecessarily Delay/Jitter which is not suitable for real time applications.

In case of Geographical routing protocol, GPSR performed well in terms of Throughput/PDR, which is slightly less, as compared to AODV and ZRP. Routing Load of GPSR is slightly less than LAR, but greater than AODV/ZRP. Jitter value for GPSR is higher than the LAR. LAR could not perform well because routing decisions also depends upon the GPS coordinates, frequent topological updates produce unnecessarily extra control overhead which leads to lowest Throughput/PDR, highest Routing Load but it also offers less Jitter and Delay because, after finding the required routes, packets can be delivered directly to the detonation.

These simulation results can be adopted to enhance the performance of ad hoc routing protocols and we can also identify the application domains as per the protocol behavior. It can be concluded that there is need to reduce the Delay and Jitter for ZRP, in order to use the real time applications over VANET. There is need to reduce the extra control overhead for AODV DSR and GPSR. Results shows that OLSR should adopt the dynamic environment by updating its route discovery and maintenance methods. LAR routing decisions are built on the basis of the location information. Frequent updates lead the extra flooding of control packets over network. If there are errors in estimated location, than it will built a inaccurate route path which can cause huge packet loss over network, so there in need to develop/update the routing logic for location estimation location error correction and which can also adopt the dynamic topology of VANETs.

\section{REFERENCES}

[1] H. El Ajaltouni, Azzedine Boukerche, Abdelhamid Mammeri, "A Multichannel QoS MAC with Dynamic Transmit Opportunity for VANets", Mobile Netw Applspringer-2013, pp.814-830

[2] J. Mun-Yee Lim, Yoong Choon Chang, Jonathan Loo, Mohamad Yusoff Alias, "Improving VANET Performance with Heuristic and Adaptive Fuzzy Logic Scheme", Wireless Perss Commun., Springer-2015, pp.

[3] Lin Zhang, Yu Liu, Zi Wang, Jinjie Guo, Yiding Huo, "Mobility and QoS oriented 802.11p MAC scheme for vehicle-to-infrastructure communications", Telecommun System-Springer-2014, pp

[4] Guangyu Li, Lila Boukhatem, Steven Martin, "An Intersection-based QoS Routing in Vehicular Ad Hoc Networks", Mobile Network App.Springer-2015, pp.

[5] V.Vijayakumara, P.Inbavallia, K.Suresh Josephb, J.Amudhavela, D.Rajaguruc, Sampath Kumard, T.Vengattaramanb, K.Premkumar, "Research on QoS aware Dynamic Reconfiguration and performance measures in VANET", GCCT, IEEE-2015, pp.829-833

[6] M. E. A. Fekair, Abderrahmane Lakas, Ahmed Korichi, "CBQoS-Vanet: Cluster-based Artificial Bee Colony Algorithm for QoS Routing Protocol in VANET", MoWNeT, IEEE-2016, pp-

[7] A. El Khatib, Azzam Mourad, Hadi Otrok, Omar Abdel Wahab, Jamal Bentahar, "A Cooperative Detection Model Based on Artificial Neural Network for VANET QoS-OLSR Protocol", IEEE-2015, pp.

[8] G. el m. ZHIOUA y, Nabil TABBANE, Houda LABIODy and Sami TABBANE, "A Fuzzy Multi-metric QoS-balancing Gateway Selection Algorithm in a Clustered VANET to LTE Advanced Hybrid Cellular Network", IEEE Transactions on Vehicular Technology2015, pp.

[9] M. H. Eiza, Thomas Owens, and Qiang Ni, "Secure and Robust Multi-Constrained QoS aware Routing Algorithm for VANETs", TDSCSI, IEEE-2014, pp.1-14 
[10] G. Rizzo, Maria Rita Palattella,Torsten Braun, Thomas Engel, "Content and Context Aware Strategies for QoS Support in VANETs", 30th International Conference on Advanced Information Networking and Applications, IEEE-2016, pp.713-723

[11] A. ENNACIRI, Mustapha MABROUKI, Mohammed Erritali, "13th International Conference Computer Graphics, Imaging and Visualization", pp-375-380

[12] Jetendra Joshi, Anshumali Singh, Lakshya Gourav Moitra and Manash Jyoti Deka, "DASITS: Driver Assistance System in Intelligent Transport System", 30th International Conference on Advanced Information Networking and Applications Workshops, pp.545-550

[13] Xin-Lin Huang, Jun Wu, Wenfeng Li,Zhifeng Zhang, Fusheng Zhu, and Minghao Wu, "Historical Spectrum Sensing Data Mining for Cognitive Radio Enabled Vehicular Ad-hoc Networks", IEEE Transactions on Dependable and Secure Computing, pp.1-13

[14] Che-Yu Chang, Hsu-Chun Yen, and Der-Jiunn Deng, "V2V QoS Guaranteed Channel Access in IEEE 802.11p VANETs", IEEE Transactions on Dependable and Secure Computing, pp.1-15
[15] B. Moussaouiz, Soufiene Djahel,Hakima Khelifiz, Salah Merni, "Towards Enhanced Reactive Routing in Urban Vehicular Ad hoc Networks", ICPE, NTDS, IEEE-2015, pp.

[16] Asif Uddin Khan, Bikram Kesari Ratha, "Time Series Prediction QoS Routing In Software Defined Vehicular Ad-hoc Network", MAMI, IEEE-2015, pp.

[17] Zubair Amjad, Wang-Cheol Song, "Road Aware QoS Routing in VANETs", APNOMS, IEEE-2015, pp.133138

[18] Doaa Al-Terri, Hadi Otrok, Hassan Barada, Mahmoud Al-Qutayri, Raed M. Shubair, Yousof Al-Hammadi, "QDSR Protocol in Vehicular Ad-hoc Networks", IIT-2015, pp.162-165

[19] http://sumo.dlr.de/wiki/Tutorials/Import_from_OpenStre etMap

[20] http://sumo.dlr.de/wiki/Tutorials/Trace_File_Generation

[21] http://sumo.dlr.de/wiki/Tools/TraceExporter\#ns2.2Fns3

[22] http://www.dlr.de/ts/en/desktopdefault.aspx/tabid9883/16931_read-41000/ 